\title{
Experimental Evaluation of Koala Scat Persistence and Detectability with Implications for Pellet-Based Fauna Census
}

\author{
Romane H. Cristescu, ${ }^{1,2}$ Klara Goethals, ${ }^{3}$ Peter B. Banks, ${ }^{1,4}$ \\ Frank N. Carrick, ${ }^{2}$ and Céline Frère ${ }^{5}$ \\ ${ }^{1}$ School of Biological, Earth and Environmental Sciences, The University of New South Wales, Kensington, NSW 2052, Australia \\ ${ }^{2}$ Centre for Mined Land Rehabilitation, Sustainable Minerals Institute, The University of Queensland, St Lucia, \\ Brisbane, QLD 4072, Australia \\ ${ }^{3}$ Department of Comparative Physiology and Biometrics, Faculty of Veterinary Medicine, Ghent University, 9820 Merelbeke, Belgium \\ ${ }^{4}$ School of Biological Sciences, The University of Sydney, Camperdown, NSW 2006, Australia \\ ${ }^{5}$ School of Land, Crop and Food Sciences, The University of Queensland, St Lucia, Brisbane, QLD 4072, Australia
}

Correspondence should be addressed to Romane H. Cristescu, romromfr@yahoo.fr

Received 1 May 2012; Revised 30 July 2012; Accepted 14 August 2012

Academic Editor: Stephen Secor

Copyright ( $) 2012$ Romane H. Cristescu et al. This is an open access article distributed under the Creative Commons Attribution License, which permits unrestricted use, distribution, and reproduction in any medium, provided the original work is properly cited.

\begin{abstract}
Establishing species distribution and population trends are basic requirements in conservation biology, yet acquiring this fundamental information is often difficult. Indirect survey methods that rely on fecal pellets (scats) can overcome some difficulties but present their own challenges. In particular, variation in scat detectability and decay rate can introduce biases. We studied how vegetation communities affect the detectability and decay rate of scats as exemplified by koalas Phascolarctos cinereus: scat detectability was highly and consistently dependent on ground layer complexity (introducing up to 16\% non-detection bias); scat decay rates were highly heterogeneous within vegetation communities; exposure of scats to surface water and rain strongly accelerated scat decay rate and finally, invertebrates were found to accelerate scat decay rate markedly, but unpredictably. This last phenomenon may explain the high variability of scat decay rate within a single vegetation community. Methods to decrease biases should be evaluated when planning scat surveys, as the most appropriate method(s) will vary depending on species, scale of survey and landscape characteristics. Detectability and decay biases are both stronger in certain vegetation communities, thus their combined effect is likely to introduce substantial errors in scat surveys and this could result in inappropriate and counterproductive management decisions.
\end{abstract}

\section{Introduction}

Knowledge of species abundance and distribution must underpin rational conservation and management decisions [1]. However, acquiring such critical information is far from a trivial undertaking $[2,3]$. This is particularly true for cryptic animals (especially when they occur at low densities) [4], for which there is often a need to use indirect survey methods [5]. These indirect methods include, but are not limited to, sign surveys $[6,7]$; of which scat (fecal pellet) survey is one of the oldest and most widely used indirect methods $[8,9]$. More specifically, scat surveys are often used to determine habitat preferences and predict habitat quality (e.g., $[10,11])$ and are thus commonly used for monitoring endangered wildlife [12] or managing game species [13]. However, variability of both scat detectability and decay rate has led to the expression of concerns regarding the reliability of such surveys [14].

Some sources of biases due to detectability and decay have been widely studied, and methods have been developed to compensate for them. For instance, scat detectability varies between observers but can be standardized by developing personal correction factors or eliminated by using the same observer [15]. Scat decay can vary widely between seasons 
and can generally be reduced by restricting the performance of surveys to certain periods of the year $[16,17]$. One critical source of bias seems unavoidable, however: environmental heterogeneity is present to some degree within almost all study sites (e.g., vegetation types, microclimate, presence and density of decomposers). The significance of the extent to which variability of environmental factors within study sites can influence scat detectability and decay rate remains unresolved [5]; thus since the occurrence of such environmental heterogeneity cannot be avoided, it is critical that the variability in scat detectability and decay rates between environments is quantified and accounted for.

Koalas, Phascolarctos cinereus, provide a good model to investigate whether and how different environmental factors influence scat detectability and decay rate. Koalas use a variety of environments (vegetation communities, exposure, soil types, etc.) and are difficult to survey directly because of their cryptic, nocturnal habits and their low population density. Scat surveys have thus been widely used in studies of koala distribution $[18,19]$, habitat use [20, 21], and abundance [22], as well as frequently forming the basis for management [23]. One source of inaccuracy reported in other species is variability of scat decay linked with diet variability [24]; this should be negligible for koalas which are folivores with a relatively homogeneous diet all year round, relying mainly on a few genera in the Family Myrtaceae, predominantly Eucalyptus, Corymbia, Melaleuca and Lophostemon [25, 26]. Thus environmental sources of variability in scat decay rates can be examined without the confounding effects of changes in diet.

Previous reports have identified the need to incorporate consideration of how koala scat decay rate [28] and detectability [22] influence the interpretation of scat surveys. Rhodes et al. [28] focused on seasonal and geographical differences in climate, which they found significantly influenced the rate of scat decay but also emphasized that most of the observed variation in scat decay rates remained unexplained and highlighted the need for more research. Robust scat survey methodology is particularly critical because land use planning and management decisions often rely on such surveys (e.g., [21, 29]). These decisions affect localized extinction risk [30] and the fate of a species is often determined by the sum of these local extinctions [31]. Such management decisions will especially impact on fauna that are not well represented in reserves due to competing land use priorities, such as fauna favoring land that is level and/or at low elevation and/or composed of highly productive soils [32]. Land use management agencies thus have a key role in species conservation [33], and they must, therefore, be confident that survey methodologies underpinning their decisions are reliable.

In this study, we conducted three experiments to investigate the effect of different environments on detectability and decay rate of koala scats. Firstly, the influence of ground layer complexity on scat detectability was investigated. Next scat decay rates in different vegetation communities used by koalas were analyzed, as was the relative influence on decay rate of local environmental variables identified from the literature as potentially important. Finally decay rates

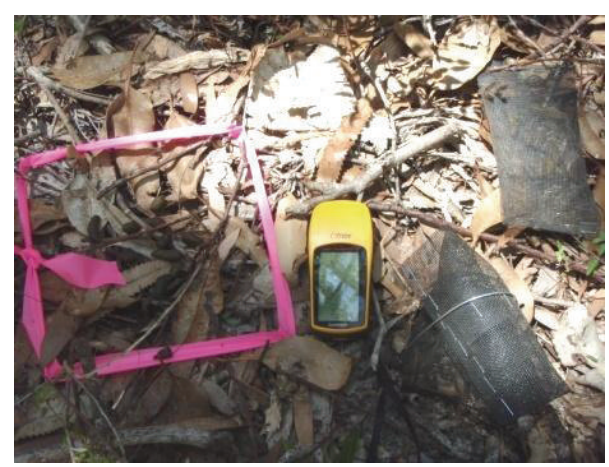

(a)

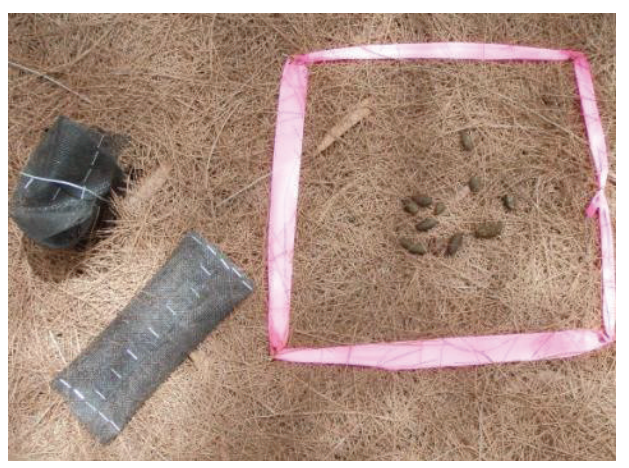

(b)

FIGURE 1: Example of two sites showing plot, cap, and bag, on two different ground layer types. (a) Complex three-dimensional litter, (b) simple Allocasuarina sp. litter.

were compared between scats protected or unprotected from the decomposing action of invertebrates.

\section{Materials and Methods}

2.1. Study Site. The field work was conducted on North Stradbroke Island (NSI), Australia $\left(27^{\circ} 23^{\prime} / 27^{\circ} 45^{\prime} \mathrm{S}\right.$, $153^{\circ} 23^{\prime} / 153^{\circ} 33^{\prime} \mathrm{E}$ ) which has an area of approximately 27,500 ha. Koalas occupy a mosaic of vegetation communities (classification based on regional ecosystems [27]) found on the island. Six remnant regional ecosystems were selected on the basis of their use by koalas on NSI [34]: mallee Eucalyptus spp. low woodland; wetlands containing Eucalyptus, Lophostemon and Melaleuca spp.; Corymbia spp. open to low closed forest; E. racemosa woodland; Melaleuca quinquenervia open forest to woodland; E. pilularis open forest. In addition, two disturbed vegetation communities (mine rehabilitation) were differentiated: one characterized by a complex litter layer, the other by a simple litter layer (see full description below and Figure 1). Three experiments were conducted as described below.

\subsection{Experiment 1: Fecal Pellet Detectability in Litter Layers of} Different Complexities. In order to measure variation in the detectability of scats associated with vegetation communities of varying ground layer complexity, 30 plots $(1 \times 5 \mathrm{~m})$ were established. One researcher dispersed a random number of 
scats (1-22) in each plot. We used old scats collected from the field, as fresh pellets have a more conspicuous color and patina that makes them easier to find. This fresh condition lasts only a few days, consequently it does not characterize most scats naturally found during surveys (RC, personal observation). A second researcher (RC), who conducted all searches to eliminate observer bias [15], searched the plot without prior knowledge of the number of scats in the plot (zero scat was a possible outcome). The search had no time limit and ended when the second researcher was confident the plot had been thoroughly searched. The total time taken to search the plot to achieve this level of confidence was recorded, as well as the percentage of scats found and the time needed to find each scat.

Plots were established in vegetation communities classified in three groups on the basis of ground layer complexity: (1) the simple litter group $(N=10)$ which had a flat litter layer composed of Allocasuarina needles, with little to no plants or woody debris $(<5 \%)$ and varying amounts of bare ground ( 0 to $20 \%)$; (2) the complex litter group $(N=10)$ which was composed of a three-dimensional litter of leaves and bark, no bare ground, and some plants and woody debris (between $20 \%$ and $90 \%$, Figure 1); (3) the highly complex litter group $(N=10)$, in which the substrate was mostly covered with plants and woody debris $(>90 \%)$. For the simple and complex litter groups, all plots were searched for scats beforehand to ensure that no scat was present prior to the experiment. For the highly complex litter group, however, presearching the plots would have disturbed the plots to an extent that would have compromised the experiment; thus they were not presearched. In order to minimize unwanted presence of scats prior to our experiment in the highly complex plots, we located the plots outside the known koala distribution on the island but with substrates comparable to the highly complex litter substrates in areas known to be used by koalas. Each of the three litter groups was replicated at two locations (five plots at each location). At each location, plots were placed $50 \mathrm{~m}$ apart from one another.

\subsection{Experiment 2: Scat Decay Rate in Different Vegetation} Communities. Fresh scats (1,980 in total) were collected from 15 females and 31 males aged from 1 to 10 years and housed at the Australian Wildlife Hospital; these were placed in different vegetation communities, and their decay rate was recorded. The koalas had been in the hospital for less than 2.5 months, and none had received treatments that could alter scat decay rate (e.g., worm treatment). Hospital cages are cleaned daily so all scats used in this experiment were less than $24 \mathrm{~h}$ old. Scats from all 46 koalas, were mixed and ten scats were randomly selected to form a group. Each scat group was weighed and groups $<6 \mathrm{~g}$ or $>10 \mathrm{~g}$ were discarded to ensure homogeneity of groups.

On 14 and 15 February 2010 (scats were stored in a refrigerator overnight), scat groups were placed in each of the eight different vegetation communities previously described, with three replicates per vegetation community (24 locations in total). Replicates were several kilometers apart $($ mean $=6.6 \mathrm{~km}, \mathrm{SD}=3.9)$ and at each location, two pseudo replicates (50 $\mathrm{m}$ apart) were laid, amounting to 48 sites in total. Each scat group was placed in a $10 \times 10 \mathrm{~cm}$ plot on the ground directly below the canopy of potential koala fodder or roosting trees of genera Eucalyptus, Corymbia, Melaleuca, or Lophostemon.

During the first 48 hours after scat placement, the study sites were struck by unusually heavy rainfall. Sites were checked the next day and some scats had already disappeared. It was feared that the rain had either soaked and disintegrated some scats or washed them away. Consequently, 10 randomly selected new scats were added in an additional plot next to each of the initial 48. Hereafter, the initial plots will be referred to as rained plots and the ones deployed just after the rain will be simply referred to as plots. High rainfall events were not recorded again during the rest of the experiment.

Scat locations were visited once a week, when the number and condition of remaining scats were recorded and the condition of scats described (from intact to scat almost unrecognizable, Table 1). When the weekly observations indicated decay rate had slowed down, scats were checked every two weeks, then less often. The survey lasted 36 weeks in total, by which time $50 \%$ of the scats had disappeared. Again, counts and classifications of the condition of scats were conducted by a single researcher to eliminate observer bias [15].

Variables characterizing the local environment in which the scats were observed were also recorded at each visit. Scat moisture (wet or dry), accumulation of litter fallen on top of scats (presence/absence), and the activity of detritivores (defined here as the presence/absence of invertebrates or fungal-type organisms) on scats were recorded as binary scores. At the end of the experiment the results were averaged. Site elevations were extracted using Terramodel Version 10.61 from a 2008 airborne laser scan of the island (Sibelco, unpublished data). A final environmental variable was flooded. The flooded variable indicated, for individual plots, the occurrence of surface water in the vicinity of scats at any time during the experiment.

Although, as indicated above, in some species scat decay rate can vary with diet, we did not expect that scats from hospitalized koalas would decay significantly differently from scats of free-ranging koalas. Indeed, the food items consumed were closely related, even if differences in the particular tree species eaten occurred (Table 2). However, we tested this assumption by comparing the decay rate of scats from hospitalized koalas and free-ranging koalas from NSI. Scats from free-ranging koalas were collected from two females. Groups of ten randomly selected scats were deposited beside plots representing six out of the eight vegetation communities previously described, since it was not possible to collect sufficient fresh scats from wild koalas to include all eight communities.

2.4. Experiment 3: Variability of Scat Decay Rate in Relation to Invertebrates. Lepidopteron larvae develop in and consume koala scats, while adult Coleoptera also exploit koala scats as a food source [35-37]. To investigate the effect of invertebrates on scat decay rate, we partially or totally 
TABLE 1: Condition of scats at Week 1 and Week 12 in the different treatments.

\begin{tabular}{|c|c|c|c|c|c|c|c|c|c|c|c|}
\hline \multirow{2}{*}{ Treatments } & \multicolumn{7}{|c|}{ Week 1} & \multicolumn{4}{|c|}{ Week 12} \\
\hline & Intact & Fibrous & Surface eaten & Part eaten & Mass of fibre & Melted & Buried & Part eaten & Half eaten & Fibrous & Melted \\
\hline Plot & $270^{* *}$ & $107^{* *}$ & $32^{*}$ & $63^{* *}$ & $1^{*}$ & 0 & 7 & $220 * *$ & $114^{* *}$ & $25^{* *}$ & 0 \\
\hline ঠ் Rained plots & $132^{* *}$ & $129^{* *}$ & $44^{*}$ & $113^{* *}$ & $18^{*}$ & 5 & 0 & $165^{* *}$ & $118^{* *}$ & $19^{* *}$ & 0 \\
\hline 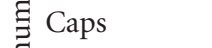 & $217^{* *}$ & $72^{* *}$ & $27^{*}$ & $93^{* *}$ & $4^{*}$ & 1 & 9 & $241^{* *}$ & $111^{* *}$ & $3^{* *}$ & 4 \\
\hline$\stackrel{\Xi}{\approx}$ Bags & $272^{* *}$ & $183^{* *}$ & $6^{*}$ & $14^{* *}$ & $2^{*}$ & 1 & 0 & $454^{* *}$ & $23^{* *}$ & $1^{* *}$ & 1 \\
\hline 凹ூ Test statistics & 32.1 & 17.6 & 14.3 & 43.1 & 14.3 & 6.2 & 7.5 & 88.6 & 44.9 & 16.5 & 2.0 \\
\hline$P$ values & $<0.001$ & 0.001 & 0.002 & $<0.001$ & 0.002 & 0.102 & 0.057 & $<0.001$ & $<0.001$ & 0.001 & 0.570 \\
\hline$\approx$ Plot & 56.3 & 22.3 & 6.7 & 13.1 & 0.2 & 0 & 1.5 & 61.3 & 31.8 & 7.0 & 0 \\
\hline$\stackrel{\text { I }}{=}$ Rained plots & 29.9 & 29.3 & 10.0 & 25.6 & 4.1 & 1.1 & 0.0 & 54.6 & 39.1 & 6.3 & 0 \\
\hline$\underset{\widetilde{J}}{\breve{d}}$ Caps & 51.3 & 17.0 & 6.4 & 22.0 & 0.9 & 0.2 & 2.1 & 67.1 & 30.9 & 0.8 & 1.1 \\
\hline A Bags & 56.9 & 38.3 & 1.3 & 2.9 & 0.4 & 0.2 & 0.0 & 94.8 & 4.8 & 0.2 & 0.2 \\
\hline
\end{tabular}

Scat number: in bold, results are significantly different across treatments, Kruskal-Wallis tests $(d f=3)$ : ${ }^{*} P \leq 0.001,{ }^{*} P<0.01$.

Condition of scats are classified as (1) intact: the scat was complete; (2) surface eaten: the scat presented a rough surface; (3) partly eaten: parts of the scat were missing; (4) half eaten: at least half of the scat had disappeared; (5) fibrous: the inner matrix had disappeared, only fibers remained visible, but the scat shape remained present; (6) mass of fiber: scats constituted of a shapeless mass of fiber. Rare states of scats were (7) scats "melted" to a shapeless entity or (8) partially buried by invertebrates.

TABLE 2: Eucalypt species fed to hospitalised koalas between 1 and 6 days [26] before their scats were collected in comparison to species found in the diet of wild koalas on NSI (only E. tereticornis, E. robusta, and E. resinifera are present on NSI, their percentages are taken from Cristescu et al. [34]).

\begin{tabular}{|c|c|c|c|}
\hline Common name & Scientific name & $\%$ fed between last 1 to 6 days & \% eaten by NSI wild koalas \\
\hline Grey gum & E. propinqua, E. punctata, E. major & $41 \%$ & \\
\hline Blue gum & E. tereticornis & $23 \%$ & $12 \%$ \\
\hline River red gum & E. camaldulensis & $17 \%$ & \\
\hline White gum & E. dunnii & $5 \%$ & \\
\hline Swamp mahogany & E. robusta & $5 \%$ & $4 \%$ \\
\hline Flooded gum & E. grandis & $4 \%$ & \\
\hline Red stringybark & E. resinifera & $2 \%$ & $11 \%$ \\
\hline Ironbark & E. crebra & $1 \%$ & \\
\hline Mountain Blue gum & E. deanei & $1 \%$ & \\
\hline Spotted gum & E. maculata & $1 \%$ & \\
\hline
\end{tabular}

protected some scats from their influence. Next to the 48 plots described earlier, two groups of 10 random scats were added (Figure 1). One group of 10 scats was placed on the ground, covered with an insect screen $(1 \mathrm{~mm}$ mesh, which was expected to be fine enough to impair lepidopteron and coleopteran access) secured into the ground to protect the scats from ground-surface-dwelling and flying invertebrates. These protected scat groups are referred to as caps. The second groups of 10 scats (referred to as bags) were placed into a sealed insect screen bags and placed directly on the litter. This protected the scats from any invertebrate damage (i.e., no invertebrate was observed inside the bags during any visits, while some invertebrates were recorded on the scats in the plots, see Experiment 2). All the scats in caps and bags were deposited prior to the heavy rainfall event. Scats in caps and bags were checked at Week 1 and Week 12 and the numbers of scats remaining and their condition were compared to the unprotected scats in the rained-plots and plots. Scats in caps and bags were observed to be wet and thus, as anticipated, the treatments did not seem to have protected the scats from the rain.
2.5. Data Analysis. All variables were tested for normality and homoscedasticity (Levene's test) and appropriate parametric or nonparametric tests were performed in PAWS Statistics 18.0 [38]. Significance was taken to be $P<$ 0.05 (except when accounting for Bonferroni's adjustment), standard error of mean (SEM) and standard deviation (SD) are given as appropriate [39].

Relationships between response and explanatory variables were investigated using a survival model for intervalcensored data [40], with the 48 sites treated as random effect (more often referred to as a frailty effect in survival analysis, $[41,42])$. Our data were analyzed using the proportional hazards model proposed by Bellamy et al. [41]. A Weibull distribution [43] was assumed for the event times and a lognormal distribution for the frailties [41].

For model selection, an a priori model approach was used so as to avoid data dredging $[44,45]$ and to reduce the possible selection of noise variables [46]. A priori models were chosen to investigate the main variable of interest (vegetation communities) and to see if any other environmental variables had an additional effect, while taking 
TABLe 3: Percentage of scats detected and time needed to detect them, in three different ground layer complexities.

\begin{tabular}{lccccccc}
\hline $\begin{array}{l}\text { Characteristics of } \\
\text { search }\end{array}$ & $\begin{array}{l}\text { Mean percentage of } \\
\text { scats found }( \pm \text { SEM) }\end{array}$ & \multicolumn{2}{c}{$\begin{array}{c}\text { Time of search (min) } \\
\text { Mean }\end{array}$} & $\begin{array}{c}\text { Mean time taken } \\
\text { by scat }(\mathrm{sec})\end{array}$ & $\begin{array}{l}\text { Mean percentage } \\
\text { found at 2 min }\end{array}$ & $\begin{array}{l}\text { Mean time to find } \\
\text { the 1st scat }(\mathrm{sec})\end{array}$ \\
\hline Simple litter & 100.0 & 3.4 & 2.1 & 6.2 & 0.73 & 87.3 & 22 \\
Complex litter & $97.7( \pm 1.1)$ & 15.8 & 7.1 & 25.2 & 1.05 & 30.3 & 10.1 \\
Highly complex litter & $83.9( \pm 4.4)$ & 34.3 & 15.4 & 48.6 & 2.61 & 11.2 & 17.5 \\
\hline
\end{tabular}

SEM: standard error of mean.

into account the known effect of rain [47]. Environmental variables chosen based on the literature $[17,48,49]$ were: detritivores, litter, elevation, and flooded (scat moisture was measured but not included, as it was correlated with other explanatory variables). Models were based on the two main variables (vegetation communities and rain) separately or in combination, then each combination of the four other environmental variables was added.

Data were graphically analyzed for skewed explanatory variables and none was observed. Explanatory variables were standardized ( $z$-transformed) to allow comparisons of model parameter estimates [50] and collinearity was tested with a variance inflation factor (VIF). As reported above, moisture level had to be excluded as it was correlated with other explanatory variables. Models were fitted with the nlmixed procedure in SAS 9.2 for Windows and were ranked on the basis of AICc, the Akaike's information criterion corrected for small sample size.

Multimodel inference methods were used to determine the relative importance of explanatory variables based on our set of models. Based on AICc, Akaike differences $(\Delta)$ between each model and the most parsimonious model were calculated, as well as Akaike weights, a measure of the weight of evidence of each model; and the evidence ratios. To account for model uncertainty, the model average parameter estimates and the unconditional standard error of each estimate were calculated.

\section{Results}

3.1. Experiment 1: Fecal Pellet Detectability in Litter Layers of Different Complexities. The percentage of scats found decreased with increased litter complexity (Kruskal-Wallis ${ }_{2}=$ $14.85, P=0.001)$; from $100 \%$ of scats found in simple litter to $97.7 \%(\mathrm{SEM}=1.1)$ in complex litter and down to $83.9 \%$ $(\mathrm{SEM}=4.4)$ in highly complex litter (Table 3$)$. The total time taken to search the plot increased with litter complexity (Kruskal-Wallis ${ }_{2}=24.56, P<0.001$ ). It took an average of 3.4 minutes $(\mathrm{SD}=1.2)$ to search plots in simple litter, 15.8 minutes $(\mathrm{SD}=6.6)$ in complex litter, and up to 34.3 minutes $(\mathrm{SD}=10.0)$ in highly complex litter. The mean time taken to find a scat increased with litter complexity (Kruskal-Wallis 2 $=16.29, P<0.001$ ), while the mean percentage of scats found after searching for $2 \mathrm{~min}$ decreased with litter complexity (Kruskal-Wallis $2=23.06, P<0.001$ ). However, the mean time to find the first scat was similar across the three litter complexities (Kruskal-Wallis $2=0.01, P=0.995$ ).

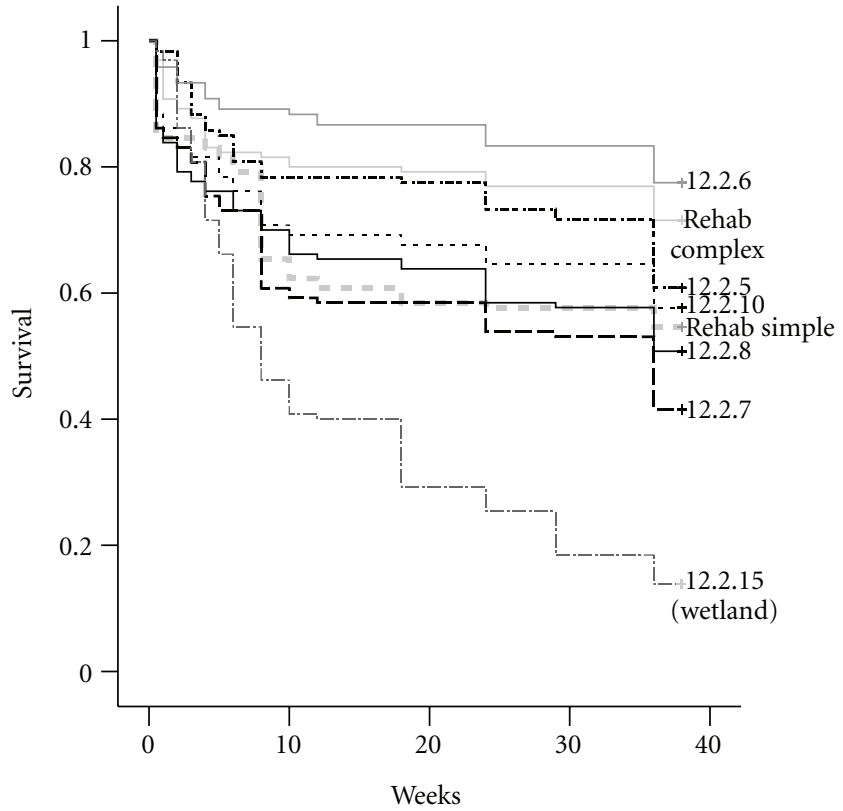

FIGURE 2: Kaplan-Meier survival curves of scats, displayed by vegetation community. NB: vegetation communities (12.2.10: mallee Eucalyptus spp. low woodland, 12.2.15: wetlands, 12.2.5: Corymbia spp. open to low closed forest, 12.2.6: E. racemosa woodland, 12.2.7: Melaleuca quinquenervia open forest to woodland, and 12.2.8: E. pilularis open forest) follow Queensland Herbarium [27] except for the addition of simple and complex rehabilitation.

3.2. Experiment 2: Scat Decay Rate in Different Vegetation Communities. Before performing any analyses, we confirmed that the average weight of each group of 10 scats was not different between and within vegetation communities (ANOVA: rained-plots: $F_{7,47}=1.007, P=0.441$; plots: $F_{7,47}$ $=2.107, P=0.065)$. Next it was confirmed that the origin of scats (free-ranging/hospitalized koalas) had no significant effect on scat decay rate (survival model, $\beta=-0.14$; $\mathrm{SE}=$ $0.20 ; P=0.51)$.

Survival curves [51] showed that scats placed in wetlands decayed faster than scats placed in any other vegetation community investigated (Figure 2). Within each vegetation community, the number of scats remaining after 36 weeks was highly variable (every possibility between zero and 10 scats, Figure 3(a)). When averaged across the 36 weeks duration of the experiment, all vegetation communities had a median of between 7 and 9 remaining scats, except wetlands which had a median of 5 (Figure 3(b)). 
The most parsimonious survival model contained rain, flooded, and litter (Table 4). This model indicated that both rain and flood increased scat decay rate and that litter decreased decay rate (Table 5); however, four of the 48 models were well supported $(<2 \Delta \mathrm{AIC})$. The three variables characterizing the most parsimonious model were also present in all other models within $2 \Delta \mathrm{AIC}$. The "vegetation" variable was not incorporated in the four best models and only one vegetation type was associated with a significant influence on scat decay in our study: wetlands were found to increase decay rate (Table 5). It is noteworthy that most plots in wetlands had been consistently flooded (all but one of the wetland plots was flooded, whereas only one plot outside wetlands, in Melaleuca quinquenervia open forest to woodland, was flooded).

\subsection{Experiment 3: Variability of Scat Decay Rate in Relation to} Invertebrates. After one week, the number of scats remaining in the rained plots was similar to the number of scats protected from invertebrates by caps placed above them (Mann-Whitney test $=932.0, P=0.077$ ); both were lower than the number of scats protected in bags (see Table 6 for details). After 12 weeks, rained plots contained significantly fewer scats than found in caps, which in turn contained fewer scats than remained in bags (see Table 6 for values and $P$ values). The condition of the scats also varied with the treatments; with the best preserved scats being the ones in the bags, while the most deteriorated scats were in the rained plots (Table 1). Overall, the scats protected from all invertebrate activity in bags were best preserved in terms of both quantity and quality, while scats in the rained plots were the worst preserved on both accounts.

\section{Discussion}

4.1. Scat Detectability and Bias. Most study sites encompass different vegetation types with potentially variable ground layers. Here we demonstrated that scat detectability varied with ground layer complexity, with up to $16 \%$ variation in the proportion of scats detected between plots of different ground layer complexities. The measured $16 \%$ variation in scat detectability most likely underestimated the bias ratio that would be present in many other scat survey studies: (a) the time dedicated to searching for scats (up to 48 minutes) was much higher in our study than would be provided for in most scat surveys which usually have to be performed under arbitrary time and budget constraints; (b) no maximum time limit was imposed for scat searches; (c) limited search time would most likely decrease the probability of finding scats, especially at sites with high ground layer complexities. Nevertheless, the majority of studies relying on scat surveys for koalas use some variation of the Spot Assessment Technique (SAT) $[52,53]$, where the search for scats lasts for a maximum of 2 minutes (or until the first scat is found). Our results demonstrate that with a constant amount of search time per tree, proportionately more scats would be missed in complex ground layers and thus a detectability bias would be introduced. Given this potential detectability bias, improving

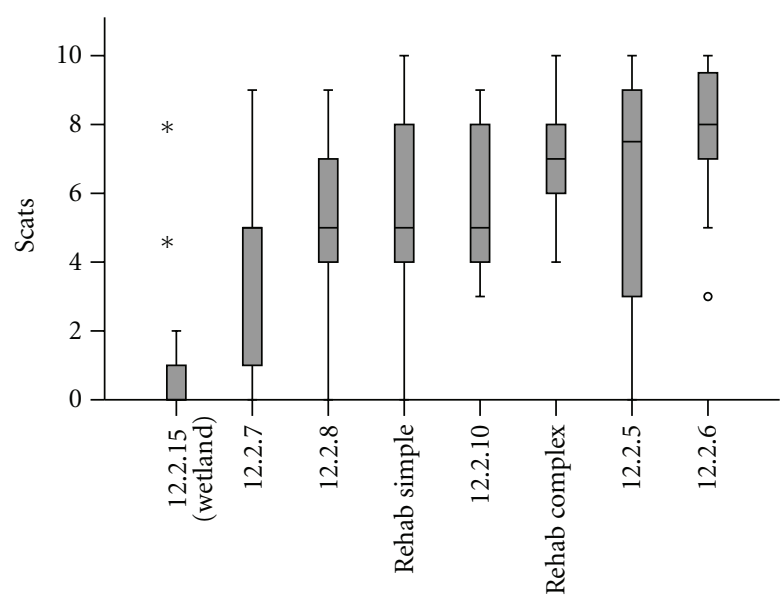

(a)

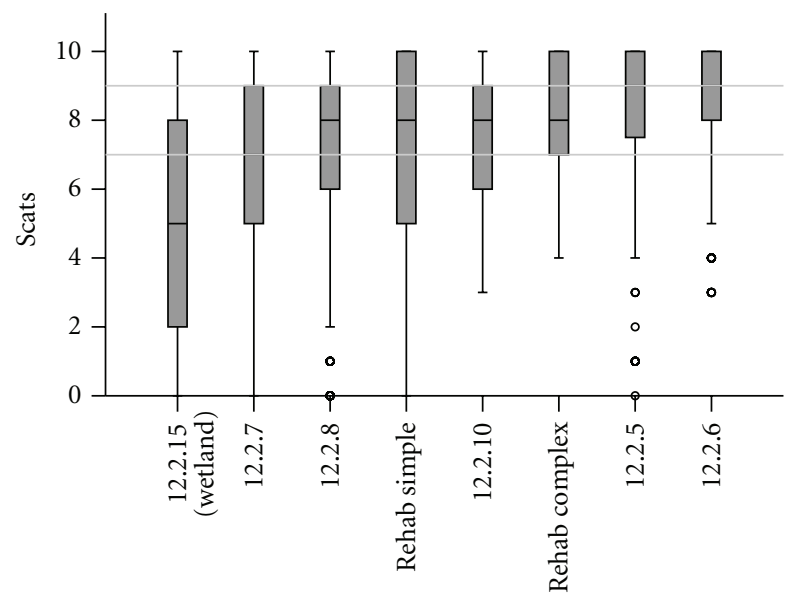

(b)

FIGURE 3: Boxplots (minimum, first quartile, median, third quartile, and maximum, with outliers $\circ$ and extreme values $*$ ) show-ing the variability of the average number of scats remaining by vegetation community (a) after 36 weeks, (b) all 36 weeks combined (grey lines in (b) include max and min medians of all but wetlands). Boxplots are ordered from the vegetation community with the least remaining scats (12.2.15) to the one with most remaining scats (12.2.6). NB: vegetation communities (12.2.10: mallee Eucalyptus spp. low woodland, 12.2.15: wetlands, 12.2.5: Corymbia spp. open to low closed forest, 12.2.6: E. racemosa woodland, 12.2.7: Melaleuca quinquenervia open forest to woodland, and 12.2.8: E. pilularis open forest) follow Queensland Herbarium [27] except for the addition of simple and complex rehabilitation.

the robustness of scat surveys requires standardizing the search area (plots of a fixed size) rather than standardizing search time.

This detectability limitation might be lessened because the SAT search is terminated when the first scat is found. In all the plots in our study, the first scat was found within the first two minutes of search, and the time to find the first scat appeared not to be correlated with the complexity of the substrate. Nonetheless, it is likely that the search time to find the first scat would be increased in many real-world situations, in contrast to our survey sites where scats were 
TABLE 4: Model selection for explaining scat decay rate during 36 weeks, all models were fitted with interval censored survival model with the 48 sites as frailty effect.

\begin{tabular}{|c|c|c|c|c|}
\hline Models & $\mathrm{AICc}$ & $\triangle \mathrm{AICc}$ & AICc weight & Evidence ratio \\
\hline Rain + litter + flooded & 3633.4 & 0 & 0.259 & 1 \\
\hline Rain + litter + flooded + elevation & 3633.5 & 0.1 & 0.246 & 1.05 \\
\hline Rain + litter + detritivores + flooded + elevation & 3633.6 & 0.2 & 0.234 & 1.11 \\
\hline Rain + litter + detritivores + flooded & 3633.9 & 0.5 & 0.202 & 1.28 \\
\hline Vegetation + rain + litter + detritivores + flooded & 3639.6 & 6.2 & 0.012 & 22.20 \\
\hline Vegetation + rain + litter + detritivores + flooded + elevation & 3639.8 & 6.4 & 0.011 & 24.53 \\
\hline Rain + detritivores + flooded & 3639.9 & 6.5 & 0.010 & 25.79 \\
\hline Vegetation + rain + litter + flooded & 3640.5 & 7.1 & 0.007 & 34.81 \\
\hline Rain + detritivores + flooded + elevation & 3640.6 & 7.2 & 0.007 & 36.60 \\
\hline Vegetation + rain + litter + flooded + elevation & 3641.3 & 7.9 & 0.005 & 51.94 \\
\hline Vegetation + rain + detritivores + flooded + elevation & 3644.3 & 10.9 & 0.001 & 232.76 \\
\hline Vegetation + rain + detritivores + flooded & 3644.9 & 11.5 & 0.001 & 314.19 \\
\hline Rain + flooded & 3644.9 & 11.5 & 0.001 & 314.19 \\
\hline Vegetation + rain + litter + detritivores + elevation & 3645.3 & 11.9 & 0.001 & 383.75 \\
\hline Rain + litter + elevation & 3645.7 & 12.3 & 0.001 & 468.72 \\
\hline
\end{tabular}

AICc: akaike information criterion corrected for small sample size, $\triangle$ AICc: AICc differences, see descriptions in text (models with a weight $<0.001$ are not shown).

TABLE 5: Relative variable importance (by decreasing importance) across all models searching for environmental variables explaining koala scat decay.

\begin{tabular}{lccc}
\hline Variable & Model-averaged estimate & Unconditional standard error estimator & Relative variable importance \\
\hline Rain & 1.02 & 0.26 & 0.99 \\
Flooded & 0.67 & 0.10 & 0.99 \\
Litter & -1.06 & 0.35 & 0.98 \\
Elevation & -0.01 & 0.00 & 0.50 \\
Detritivores & -0.97 & 0.72 & 0.48 \\
Rehabilitation & -0.10 & 0.47 & 0.03 \\
RE 12.2.5 & 0.55 & 0.57 & 0.03 \\
RE 12.2.6 & 0.14 & 0.56 & 0.03 \\
RE 12.2.7 & 0.12 & 0.41 & 0.03 \\
RE 12.2.8 & 0.56 & 0.42 & 0.03 \\
RE 12.2.10 & 0.45 & 0.48 & 0.03 \\
RE 12.2.15 & 0.94 & 0.60 & 0.03 \\
\hline
\end{tabular}

TABLE 6: Number of scats remaining at week 1 and week 12 in the different treatments $(N=48$ per treatment, see text $)$ and statistical differences between treatments ( $P$ values and Mann-Whitney $U$ test statistics, Bonferroni's adjustment $\alpha=0.004$ ).

\begin{tabular}{|c|c|c|c|c|c|c|c|c|c|c|c|}
\hline & & \multirow{2}{*}{ Mean } & \multirow{2}{*}{ SD } & \multirow{2}{*}{ Min } & \multirow{2}{*}{ Max } & \multicolumn{2}{|c|}{ Rained plot } & \multicolumn{2}{|c|}{ Cap } & \multicolumn{2}{|c|}{ Bag } \\
\hline & & & & & & $U$ & $P$ value & $U$ & $P$ value & $U$ & $P$ value \\
\hline \multirow{4}{*}{ Week 1} & Plot & 9.85 & 0.46 & 8 & 10 & 612.5 & $<0.001$ & 845.5 & 0.002 & 1078.0 & 0.229 \\
\hline & Rained plot & 8.17 & 2.43 & 0 & 10 & & & 932.0 & 0.077 & 557.0 & $<0.001$ \\
\hline & Cap & 8.75 & 2.37 & 2 & 10 & & & & & 781.0 & $<0.001$ \\
\hline & Bag & 9.96 & 0.2 & 9 & 10 & & & & & & \\
\hline \multirow{4}{*}{ Week 12} & Plot & 7.63 & 2.66 & 0 & 10 & 680.0 & $<0.001$ & 1055.0 & 0.467 & 438.0 & $<0.001$ \\
\hline & Rained plot & 5.94 & 2.75 & 0 & 10 & & & 643.0 & $<0.001$ & 93.5 & $<0.001$ \\
\hline & Cap & 7.67 & 3.12 & 0 & 10 & & & & & 575.5 & $<0.001$ \\
\hline & Bag & 9.75 & 0.93 & 5 & 10 & & & & & & \\
\hline
\end{tabular}

Min: minimum number of scats, max: maximum number of scats. 
deposited just before the search (for example, this precluded the pellets being obscured by litter fall). Perhaps even more importantly, a major component of the present study was to investigate the recovery rate of scats from different substrates, thus the numbers of scats deposited in the plots were higher than would be found naturally in most circumstances. From the data collected from 36 plots on North Stradbroke Island [54], the average natural scat density was just under 1,100 per hectare, whilst in the experimental plots in this paper, the (artificial) scat density was over 27,300 per hectare. Thus the time to find the first scat during searches could potentially be around 25 times longer in natural conditions than in our experiment; hence it will often require much more than two minutes to find the first scat in routine surveys.

The detectability bias determined in the present study of up to $16 \%$ (likely to be even higher in the context of SATtype approaches) has major implications for indirect fauna surveys. Even slight variations in detection probability across different vegetation communities can seriously skew study results $[55,56]$. A simulation study modeling species occurrence as a function of habitat covariates evaluated the errors resulting from imperfect detections [55]. A detectability bias of 15 to $20 \%$ (as found in our study) resulted in up to $200 \%$ relative bias when estimating parameters (i.e., an over or under estimation of $200 \%$ of the effect of a habitat variable on a species' occupancy).

A widely used and efficient technique to account for imperfect detection is distance sampling, a method where perpendicular distance from a transect to a fecal mass is used to estimate a detection function [57]. Detection functions account for a greater chance of imperfect detection further away from the transect and can be derived for observers or habitats. Distance sampling has, for instance, been widely used in dung surveys of elephant Loxodonta sp. populations [58]. However, this method is not feasible for koalas given the difficulty of locating their scats: indeed, the probability of detection for koala scats does not equal one even on the transect and thus transgresses a fundamental assumption of distance sampling [57].

Other methods have been developed to deal with imperfect detection using zero-inflated processes [2, 59$61]$. These methods rely on repeated surveys over a short time $[56,62]$. While these methods are based on direct animal surveys, they should also be applicable to indirect animal surveys. Some of these approaches even allow the probability of detection to vary with characteristics of the vegetation communities being surveyed [59]. As suggested by MacKenzie [56], repeated samples could also be collected by multiple observers carrying out searches on the same plot and comparing scat detection [15], as long as interobserver variability is modeled to reduce or eliminate bias from this source.

As a last resort, consistency in detectability bias could allow for the determination and incorporation of a correction factor. If researchers and managers are using a one off scat survey, they will need to incorporate methods to account for the effects of variation in ground complexity in their protocols. For instance, in distribution models based on scat presence/absence, the results could be weighted for variable ground layer complexity based on available studies (e.g., the present study and see also [22]).

4.2. Scat Decay Rate and Bias. In comparison to scat detectability, variability in decay rates was not consistently associated with particular vegetation communities (Figure 3, Table 4). For instance, extreme outcomes were seen for two plots inside the same vegetation community after 36 weeks, despite those plots being only $50 \mathrm{~m}$ apart: one had 100\% scats remaining and the other had 0\%. Similar results were also found in a study by Rhodes et al. [28], where a high and unexplained proportion of decay rate variability was associated with the variation between plots within a site. In the current study, it is worth noting that the treatments preventing invertebrates from accessing the scats greatly influenced scat decay rate. The fine-scale variation of invertebrate densities and the stochastic chances of invertebrates finding scats could explain much of the small-scale heterogeneity of scat decay rate. Whatever the reasons, the lack of consistency in scat decay rate makes the development of a correction factor difficult. One possible way to compensate for variability in scat decay rate could be to increase the intensity of sampling within each vegetation community in order to average out the fine-scale heterogeneity $[28,63]$.

One exception to the inconsistency in decay rate relates to the vegetation community associated with wetlands. In wetlands, the decay rate was consistently faster than that observed in other vegetation communities (Figures 2 and 3 ). Plots in wetlands were consistently and selectively flooded, which was found to accelerate scat decay rate (Table 5). This suggests that strong biases could be introduced when landscapes with surface water present (or probably even very moist substrates) are being surveyed. Koalas actually tend to favor wet habitats [21] and so for this species, there is the paradoxical likelihood that there will be a low density of detectable scats present in areas supporting a relatively high density of koalas. Despite this, few studies have attempted to control for decay bias in koala scat surveys and even these studies have tried to account for scat decay by excluding scats older than a certain threshold $[20,22,64]$. Scat aging, however, has been found to be generally unreliable [48] or at best limited to highly skilled and experienced researchers [65]. Nevertheless, some species have had more objective criteria developed such as in the case of the gorilla Gorilla gorilla, for which dung age has been reliably correlated with dung pile height [12]. For species where objective criteria to age scats are not yet available, developing correction factors by measuring scat decay rates in sites where an accelerated decay rate can be expected is strongly indicated.The most useful method for developing these factors is retrospective estimation of decay rate, where fresh scats are recorded in different habitats at different times prior to the survey. At the time of the survey, all the previously marked scat locations are revisited, and the percentage of decayed scats per habitat is calculated [66]. This accounts for specific influences (e.g., temperature, precipitation) that the surveyed scats and the marked scats share (see an example for deer in [67]). A 
TABLE 7: Comparison of the percentage of predicted scat recovery in two extreme vegetation communities based on observed detectability and decay rates.

\begin{tabular}{lccccccccc}
\hline \multirow{2}{*}{ Habitat } & \multicolumn{3}{c}{ \% scats remaining due to decay } & \multicolumn{4}{c}{ \% scats found due to both biases } \\
& \% detected & \multicolumn{2}{c}{ 3 months } & \multicolumn{2}{c}{ 9 months } & \multicolumn{2}{c}{3 months } & 9 months \\
& & No rain & Rain & No rain & Rain & No rain & Rain & No rain & Rain \\
\hline Wetland & 83.9 & 40.0 & 40.0 & 18.3 & 10.0 & 33.6 & 33.6 & 15.4 & 8.4 \\
Simple litter rehab & 100.0 & 73.3 & 50.0 & 68.3 & 42.9 & 73.3 & 50.0 & 68.3 & 42.9 \\
\hline
\end{tabular}

retrospective estimate for koala scats presents two main difficulties. (1) It is recommended for the retrospective method that the scats be surveyed long enough for $90 \%$ of scats to have decayed. Given that after 9 months only $50 \%$ of our scats had disappeared, a retrospective survey for scat decay would mean going in the field, in each different habitat, for an extensive amount of time prior to the survey. (2) On each of these occasions, koalas would have to be found and fresh scats detected and marked for the study. Koalas are difficult and time consuming to find which is, of course, why indirect methods are used in the first place. However, the use of fresh scats obtained from captive or radiotracked koalas and distributed to establish a surrogate for randomly located pellets might help resolve that second difficulty. This time-consuming aspect of the retrospective scat decay analysis approach prior to the actual scat survey needs to be taken into account when evaluating and selecting between different survey options. Furthermore, as there might be considerable interannual variations in many parameters influencing scat decay (e.g., temperature, precipitation, or even insect abundance), deriving a correction from a single survey might not be appropriate to rely upon to correct for bias in subsequent surveys. Thus the time-consuming retrospective estimate of scat decay prior to each scat survey might be warranted.

Another method proposed to limit decay bias is to clear plots yearly [48]. However, we observed substantial decay heterogeneity over a period much shorter than one year. On the basis of our results, clearing plots five weeks prior to the survey could remove bias arising from heterogeneous decay rates. This method has been used in a study of macropods, where plots were cleared one month before the survey [68]. For fauna found typically at low density, however, clearing sites one month prior to surveys might result in scats not being deposited in the interval between clearing and survey. In any case, it might not be possible to remove $100 \%$ of the scats when clearing plots, resulting in further errors ([69], but see avenues for potential solutions in [28]).

\subsection{Combination of Detectability and Decay Rate Variability.} The percentages of scats that would be found in a survey after accounting for detectability and decay biases were compared between two vegetation communities (Table 7). On the one hand, wetlands have the worst detectability owing to very dense ground vegetation and the quickest decay rate owing to their proneness to flooding. On the other hand, rehabilitated vegetation communities with simple litter recorded the best detectability and a decay rate not significantly different from most other vegetation communities. For the same density of scats deposited in wetlands and in rehabilitated areas with simple litter ground layers, the proportion of scats found in a survey would vary widely (Table 7). In the worst case, after 9 months, a survey would detect $8 \%$ of the total original scats in wetlands against $42 \%$ in rehabilitated vegetation. In this example, if the actual koala utilization rate of both areas were equal, wetlands would be wrongly classified as five times less used by koalas. A bias of this magnitude, if not able to be corrected, would invalidate any interpretation of koala distribution or habitat preference. The potential for such biases to exist more generally, seriously questions the validity of scat surveys which fail to determine whether those biases are actually present.

In this paper, we present the first comprehensive study of two significant biases of fecal pellet surveys, detectability and decay of scats, which were found to be a function of the different environments present within a study site. Biases are often acknowledged and occasionally dealt with when comparing studies being undertaken in different geographical locations, climates, or seasons. On the other hand, the biases within a particular study area are still often deemed negligible and dismissed without any real evidence. However, our study demonstrates the presence of biases inherent in using indirect signs of a species' presence for assessing the species' distribution or habitat preferences, let alone confounding estimation of its abundance. Scat detectability bias can be controlled because of its consistency and the existence of appropriate methodologies, thus it should be accounted for in future scat survey studies. Scat decay bias acts in more complex ways and may be an even more problematic issue. In extreme cases, scat decay bias might erroneously decrease the estimated value of what is actually prime habitat. This was a real effect observed in our case study, where the focal species (the koala) prefers types of habitats where there is also a higher scat decay rate. Further studies to determine exactly how to use additional corrections to decrease scat decay bias to an acceptable level (e.g., sampling effort, whether retrospective estimate of decay rate can be reused) are still required [28]. But it is clear from the present study that wet and dry sites will differ significantly in scat decay rate and until similar studies have been replicated in a variety of different environments, the conservative assumption must be that there will be a high degree of site specificity of this parameter and each distinct location will require calibration (with its own retrospective estimate of decay rate).

The magnitude of potential detectability and decay biases combined could seriously impede scat survey reliability in 
any species. These biases can occur even at a relatively small scale because most study areas are heterogeneous. Such biases associated with particular habitats are likely to introduce errors in scat surveys which in turn could lead to inappropriate management decisions.

\section{Acknowledgments}

The authors wish to thank Russell Miller for continuous assistance in the field, Olivia Woosnam-Merchez for commenting on earlier drafts, and the Lone Pine Koala Sanctuary and the Australian Wildlife Hospital for providing the fresh koala scats. The authors are grateful for the very helpful comments from two anonymous reviewers that have resulted in an improved paper. The first author is supported by an Endeavour Europe Award and an Endeavour International Postgraduate Research Scholarship. Sibelco Australia-Mineral Sand provided ongoing support to this research through the provision of logistical support and access to company sites.

\section{References}

[1] IUCN, IUCN Red List Categories and Criteria: Version 3.1, IUCN Species Survival Commission, Gland, Switzerland, 2001.

[2] A. J. Tyre, B. Tenhumberg, S. A. Field, D. Niejalke, K. Parris, and H. P. Possingham, "Improving precision and reducing bias in biological surveys: estimating false-negative error rates," Ecological Applications, vol. 13, no. 6, pp. 1790-1801, 2003.

[3] J. A. Royle and J. D. Nichols, "Estimating abundance from repeated presence-absence data or point counts," Ecology, vol. 84, no. 3, pp. 777-790, 2003.

[4] M. Kéry, "Inferring the absence of a species-a case study of snakes," The Journal of Wildlife Management, vol. 66, no. 2, pp. 330-338, 2002.

[5] G. J. Wilson and R. J. Delahay, "A review of methods to estimate the abundance of terrestrial carnivores using field signs and observation," Wildlife Research, vol. 28, no. 2, pp. 151-164, 2001.

[6] K. C. Kendall, L. H. Metzgar, D. A. Patterson, and B. M. Steele, "Power of sign surveys to monitor population trends," Ecological Applications, vol. 2, no. 4, pp. 422-430, 1992.

[7] P. D. Walsh and L. J. T. White, "Evaluating the steady state assumption: simulations of gorilla nest decay," Ecological Applications, vol. 15, no. 4, pp. 1342-1350, 2005.

[8] L. J. Bennett, P. F. English, and R. McCain, "A study of deer populations by use of pellet-group counts," The Journal of Wildlife Management, vol. 4, no. 4, pp. 398-403, 1940.

[9] R. J. Putman, "Facts from faeces," Mammal Review, vol. 14, no. 2, pp. 79-97, 1984.

[10] E. Schüttler, J. T. Ibarra, B. Gruber, R. Rozzi, and K. Jax, "Abundance and habitat preferences of the Southernmost population of mink: implications for managing a recent island invasion," Biodiversity and Conservation, vol. 19, no. 3, pp. 725-743, 2010.

[11] D. Ramp and G. Coulson, "Density dependence in foraging habitat preference of Eastern grey kangaroos," Oikos, vol. 98, no. 3, pp. 393-402, 2002.

[12] H. S. Kuehl, A. Todd, C. Boesch, and P. D. Walsh, "Manipulating decay time for efficient large-mammal density estimation: gorillas and dung height," Ecological Applications, vol. 17, no. 8, pp. 2403-2414, 2007.

[13] C. C. Webbon, P. J. Baker, and S. Harris, "Faecal density counts for monitoring changes in red fox numbers in rural Britain," Journal of Applied Ecology, vol. 41, no. 4, pp. 768-779, 2004.

[14] W. M. Block, A. B. Franklin, J. P. Ward, J. L. Ganey, and G. C. White, "Design and implementation of monitoring studies to evaluate the success of ecological restoration on wildlife," Restoration Ecology, vol. 9, no. 3, pp. 293-303, 2001.

[15] D. J. Neff, "The pellet-group count technique for big game trend, census, and distribution: a review," The Journal of Wildlife Management, vol. 32, no. 3, pp. 597-614, 1968.

[16] G. Massei, P. Bacon, and P. V. Genov, "Fallow deer and wild boar pellet group disappearance in a Mediterranean area," The Journal of Wildlife Management, vol. 62, no. 3, pp. 1086-1094, 1998.

[17] A. C. Nchanji and A. J. Plumptre, "Seasonality in elephant dung decay and implications for censusing and population monitoring in South-Western cameroon," African Journal of Ecology, vol. 39, no. 1, pp. 24-32, 2001.

[18] C. A. McAlpine, J. R. Rhodes, M. E. Bowen et al., "Can multiscale models of species' distribution be generalized from region to region? A case study of the koala," Journal of Applied Ecology, vol. 45, no. 2, pp. 558-567, 2008.

[19] J. R. Rhodes, T. Wiegand, C. A. McAlpine et al., "Modeling species' distributions to improve conservation in semiurban landscapes: koala case study," Conservation Biology, vol. 20, no. 2, pp. 449-459, 2006.

[20] B. J. Sullivan, G. S. Baxter, and A. T. Lisle, "Low-density koala (Phascolarctos cinereus) populations in the mulgalands of South-West Queensland. III. Broad-scale patterns of habitat use," Wildlife Research, vol. 30, no. 6, pp. 583-591, 2003.

[21] D. Lunney, A. Matthews, C. Moon, and S. Ferrier, "Incorporating habitat mapping into practical koala conservation on private lands," Conservation Biology, vol. 14, no. 3, pp. 669$680,2000$.

[22] B. J. Sullivan, G. S. Baxter, A. T. Lisle, L. Pahl, and W. M. Norris, "Low-density koala (Phascolarctos cinereus) populations in the mulgalands of South-West Queensland. IV. Abundance and conservation status," Wildlife Research, vol. 31, no. 1, pp. 19-29, 2004.

[23] O. Woosnam-Merchez, R. H. Cristescu, D. Dique et al., "What faecal pellet surveys can and can't reveal about the ecology of koalas Phascolarctos cinereus," Australian Zoologist. In press.

[24] G. A. Cochran and H. J. Stains, "Deposition and decomposition of fecal pellets by cottontails," The Journal of Wildlife Management, vol. 25, no. 4, pp. 432-435, 1961.

[25] R. W. Martin and K. Handasyde, The Koala: Natural History, Conservation and Management, Australian Natural History Series, University of New South Wales Press, Hong Kong, China, 1999.

[26] W. Ellis, F. Carrick, P. Lundgren, A. Veary, and B. Cohen, "The use of faecal cuticle examination to determine the dietary composition of koalas," Australian Zoologist, vol. 31, no. 1, pp. 127-133, 1999.

[27] Queensland Herbarium, Regional Ecosystem Description Database (REDD), Department of Environment and Resource Management, Brisbane, Australia, 2009.

[28] J. R. Rhodes, D. Lunney, C. Moon, A. Matthews, and C. A. Mcalpine, "The consequences of using indirect signs that decay to determine species' occupancy," Ecography, vol. 34, no. 1, pp. 141-150, 2011.

[29] D. Lunney, C. Moon, A. Matthews, and J. Turbill, Coffs Harbour City koala Plan of Management. Part B, Coffs Harbour 
Koala Study, NSW National Parks and Wildlife Service, Hurstville, Australia, 1999.

[30] H. J. Preece, "Identifying hotspots for threats to koalas using spatial analysis," 2007, http://www.mssanz.org. au/MODSIM07/papers/21_s46/IdentifyingHotsp-ots_s46_Preece.pdf.

[31] G. Caughley, "Directions in conservation biology," Journal of Animal Ecology, vol. 63, no. 2, pp. 215-244, 1994.

[32] J. M. Scott, F. W. Davis, R. G. McGhie, R. G. Wright, C. Groves, and J. Estes, "Nature reserves: do they capture the full range of America's biological diversity?” Ecological Applications, vol. 11, no. 4, pp. 999-1007, 2001.

[33] D. Press, D. F. Doak, and P. Steinberg, "The role of local government in the conservation of rare species," Conservation Biology, vol. 10, no. 6, pp. 1538-1548, 1996.

[34] R. Cristescu, W. Ellis, D. de Villiers et al., "North Stradbroke Island: an island ark for Queensland's koalas population?" Proceedings of the Royal Society of Queensland, vol. 117, pp. 309-334, 2011.

[35] T. R. New, "Concluding comment: looking to the future for moths," Journal of Insect Conservation, vol. 8, no. 2-3, pp. 275276, 2004.

[36] I. F. B. Common and M. Horak, "Four new species of Telanepsia Turner (Lepidoptera: Oecophoridae) with larvae feeding on koala and possum scats," Invertebrate Taxonomy, vol. 8, no. 4, pp. 809-828, 1994.

[37] A. Melzer, M. A. Schneider, and D. Lamb, "Insects associated with the faecal pellets of the koala, Phascolarctos cinereus Goldfuss," Australian Entomologist, vol. 21, no. 3, pp. 69-70, 1994.

[38] IBM, PAWS2009, SPSS Inc., Chicago, Ill, USA.

[39] D. G. Altman and J. M. Bland, "Statistics notes: standard deviations and standard errors," British Medical Journal, vol. 331, no. 7521, article 903, 2005.

[40] R. Peto, "Experimental survival curves for interval-censored data," Journal of the Royal Statistical Society, Series C, vol. 22, no. 1, pp. 86-91, 1973.

[41] S. L. Bellamy, Y. Li, L. M. Ryan, S. Lipsitz, M. J. Canner, and R. Wright, "Analysis of clustered and interval censored data from a community-based study in asthma," Statistics in Medicine, vol. 23, no. 23, pp. 3607-3621, 2004.

[42] K. Goethals, B. Ampe, D. Berkvens, H. Laevens, P. Janssen, and L. Duchateau, "Modeling interval-censored, custered cow udder quarter infection times through the shared gamma frailty model," Journal of Agricultural, Biological, and Environmental Statistics, vol. 14, no. 1, pp. 1-14, 2009.

[43] W. Weibull, "A statistical distribution function of wide applicability," Journal of Applied Mechanics, vol. 18, pp. 293 297, 1951.

[44] K. P. Burnham and D. R. Anderson, Model Selection and Multimodel Inference, Springer, New York, NY, USA, 2nd edition, 2002.

[45] D. R. Anderson, K. P. Burnham, W. R. Gould, and S. Cherry, "Concerns about finding effects that are actually spurious," Wildlife Society Bulletin, vol. 29, no. 1, pp. 311-316, 2001.

[46] V. F. Flack and P. C. Chang, "Frequency of selecting noise variables in subset regression analysis: a simulation study," The American Statistician, vol. 41, no. 1, pp. 84-86, 1987.

[47] O. C. Wallmo, A. W. Jackson, T. L. Hailey, and R. L. Carlisle, "Influence of rain on the count of deer pellet groups," The Journal of Wildlife Management, vol. 26, no. 1, pp. 50-55, 1962.

[48] L. R. Prugh and C. J. Krebs, "Snowshoe hare pellet-decay rates and aging in different habitats," Wildlife Society Bulletin, vol. 32, no. 2, pp. 386-393, 2004.
[49] G. S. Masunga, O. Andresen, J. E. Taylor, and S. S. Dhillion, "Elephant dung decomposition and coprophilous fungi in two habitats of semi-arid Botswana," Mycological Research, vol. 110, no. 10, pp. 1214-1226, 2006.

[50] G. Quinn and M. Keough, Experimental Design and Data Analysis for Biologists, Cambridge University Press, Cambridge, UK, 5th edition, 2006.

[51] E. L. Kaplan and P. Meier, "Nonparametric estimation from incomplete observations," Journal of the American Statistical Association, vol. 53, no. 282, pp. 457-481, 1958.

[52] S. Phillips, J. Callaghan, and V. Thompson, "The tree species preferences of koalas (Phascolarctos cinereus) inhabiting forest and woodland communities on Quaternary deposits in the Port Stephens area, New South Wales," Wildlife Research, vol. 27, no. 1, pp. 1-10, 2000.

[53] S. Phillips and J. Callaghan, "The spot assessment technique: a tool for determining localised levels of habitat us by Koalas Phascolarctos cinereus," Australian Zoologist, vol. 35, pp. 774780, 2011.

[54] R. Cristescu, "Fauna recolonisation of mine rehabilitation through the example of arboreal marsupials, with a particular focus on the koala Phascolarctos cinereus," in School of Biological, Earth and Environmental Sciences, $\mathrm{PhD}$ Thesis University of New South Wales, Sydney, Australia, 2011.

[55] W. Gu and R. K. Swihart, "Absent or undetected? Effects of non-detection of species occurrence on wildlife-habitat models," Biological Conservation, vol. 116, no. 2, pp. 195-203, 2004.

[56] D. I. MacKenzie, "What are the issues with presence-absence data for wildlife managers?" The Journal of Wildlife Management, vol. 69, no. 3, pp. 849-860, 2005.

[57] S. T. Buckland, D. R. Anderson, K. P. Burnham, J. L. Laake, D. L. Borchers, and L. Thomas, Introduction to Distance Sampling, Oxford University Press, London, UK, 2001.

[58] R. F. W. Barnes, "Estimating forest elephant abundance by dung counts," in Studying Elephants, K. Kangwana, Ed., pp. 28-37, African Wildlife Foundation, Nairobi, Kenya, 1996.

[59] D. I. MacKenzie, J. D. Nichols, G. B. Lachman, S. Droege, A. A. Royle, and C. A. Langtimm, "Estimating site occupancy rates when detection probabilities are less than one," Ecology, vol. 83, no. 8, pp. 2248-2255, 2002.

[60] B. A. Wintle, M. A. McCarthy, K. M. Parris, and M. A. Burgman, "Precision and bias of methods for estimating point survey detection probabilities," Ecological Applications, vol. 14, no. 3, pp. 703-712, 2004.

[61] H. B. Stauffer, C. J. Ralph, and S. L. Miller, "Ranking habitat for marbled murrelets: new conservation approach for species with uncertain detection," Ecological Applications, vol. 14, no. 5, pp. 1374-1383, 2004.

[62] B. A. Wintle, R. P. Kavanagh, M. A. McCarthy, and M. A. Burgman, "Estimating and dealing with detectability in occupancy surveys for forest owls and arboreal marsupials," The Journal of Wildlife Management, vol. 69, no. 3, pp. 905917, 2005.

[63] D. Lunney, S. Phillips, J. Callaghan, and D. Coburn, "Determining the distribution of koala habitat across a shire as a basis for conservation: a case study from Port Stephens, New South Wales," Pacific Conservation Biology, vol. 4, no. 3, pp. 186-196, 1998.

[64] M. Hasegawa, Habitat Utilisation By Koalas (Phascolarctos cinereus) at Point Halloran, Queensland, The University of Queensland, Brisbane, Australia, 1995.

[65] B. J. Sullivan, G. S. Baxter, and A. T. Lisle, "Low-density koala (Phascolarctos cinereus) populations in the mulgalands 
of South-West Queensland. I. Faecal pellet sampling protocol," Wildlife Research, vol. 29, no. 5, pp. 455-462, 2002.

[66] S. E. Laing, S. T. Buckland, R. W. Burn, D. Lambie, and A. Amphlett, "Dung and nest surveys: estimating decay rates," Journal of Applied Ecology, vol. 40, no. 6, pp. 1102-1111, 2003.

[67] J. F. Brodie, "An experimentally determined persistence-rate correction factor for scat-based abundance indices," Wildlife Society Bulletin, vol. 34, no. 4, pp. 1216-1219, 2006.

[68] C. N. Johnson and P. J. Jarman, "Macropod studies at Wallaby Creek. VI. A validation of the use of dung-pellet counts for measuring absolute densities of populations of macropodids," Australian Wildife Research, vol. 14, no. 2, pp. 139-145, 1987.

[69] T. R. Stanley and J. A. Royle, "Estimating site occupancy and abundance using indirect detection indices," The Journal of Wildlife Management, vol. 69, no. 3, pp. 874-883, 2005. 

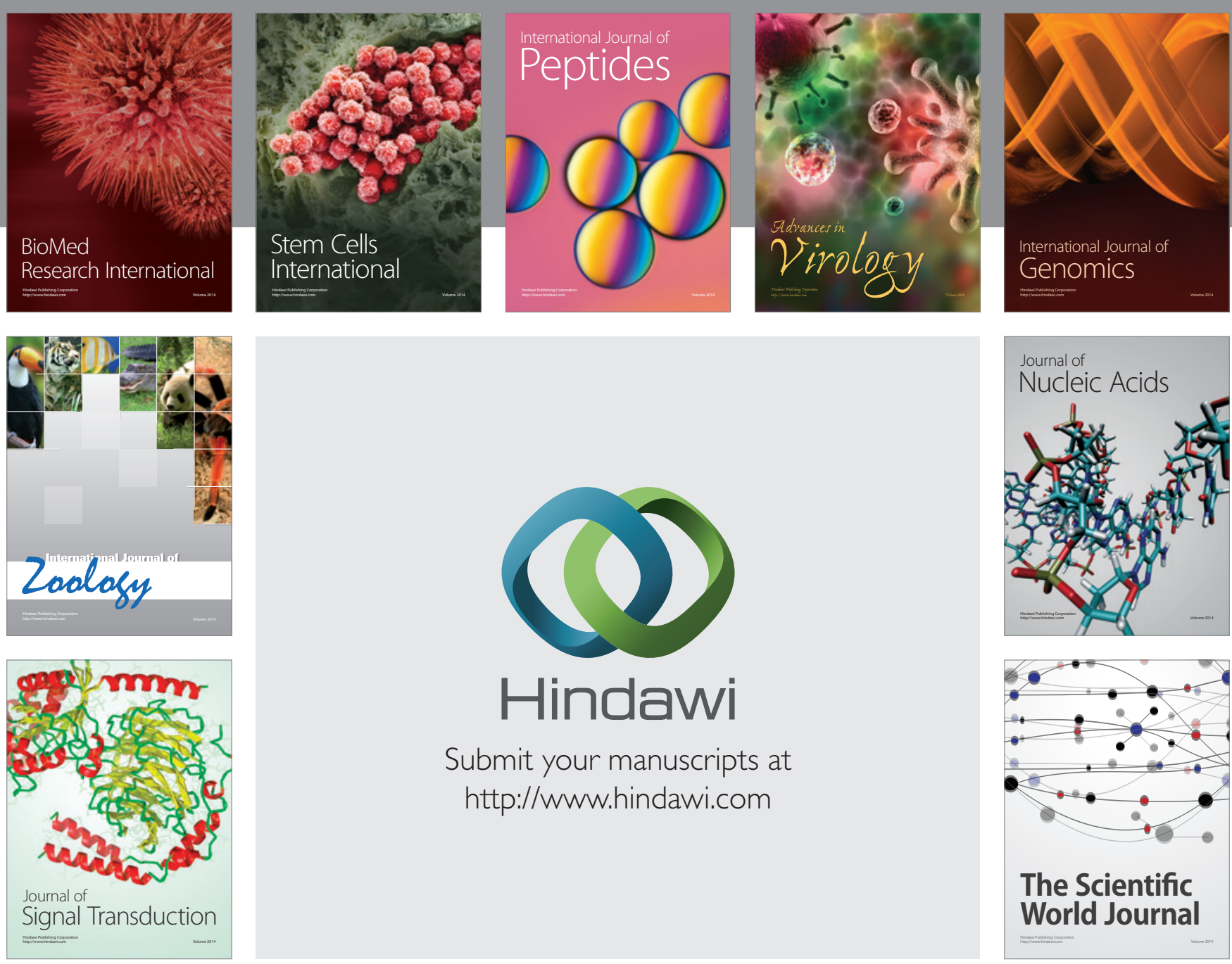

Submit your manuscripts at

http://www.hindawi.com
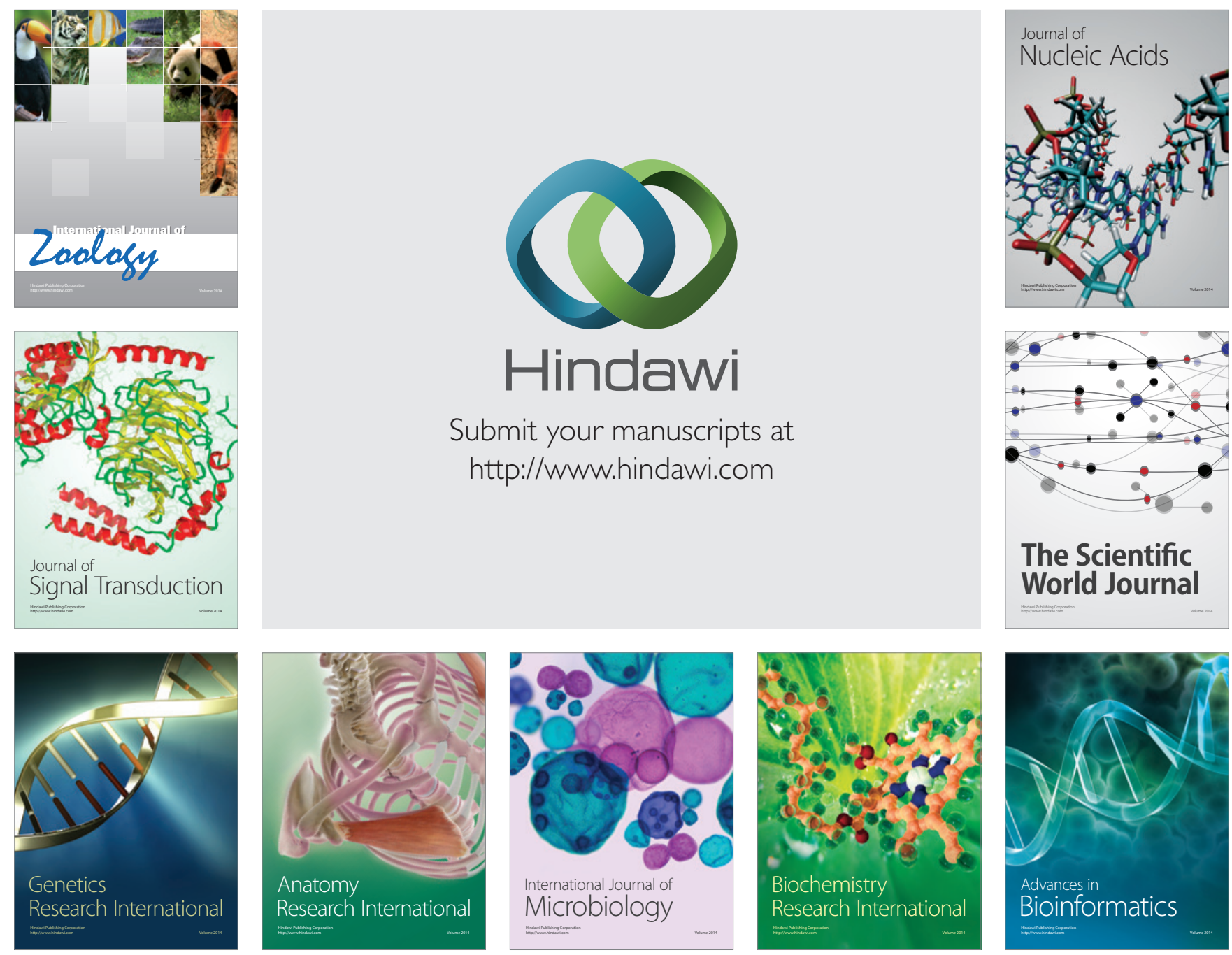

The Scientific World Journal
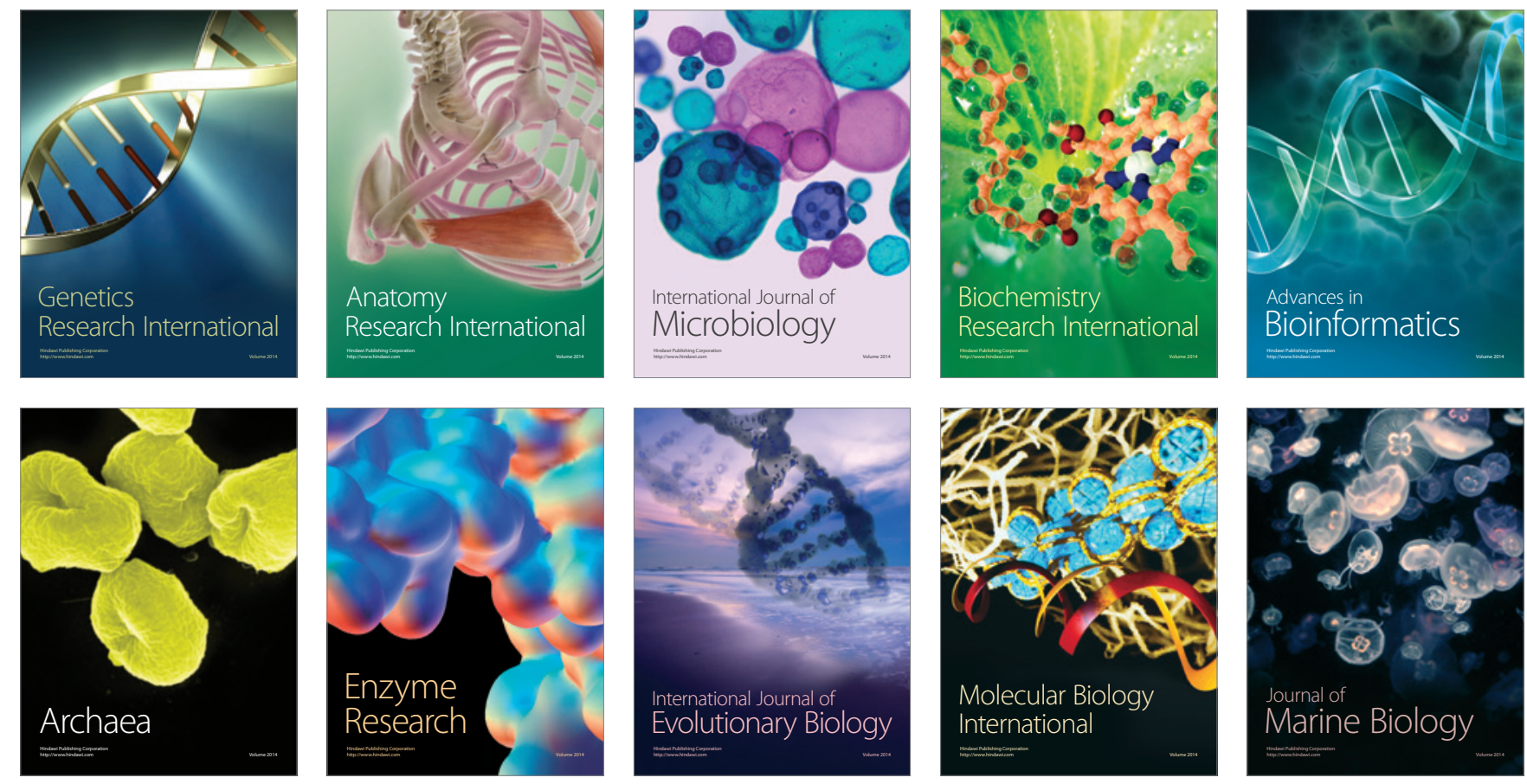